\title{
A Compact Wideband SIW Bandpass Filter with Wide Stopband and High Selectivity
}

\author{
Lei Huang * and Naichang Yuan \\ State Key Laboratory of Complex Electromagnetic Environment Effects on Electronics and Information System, \\ College of Electronic Science, National University of Defense Technology, Changsha 410073, China; \\ Yuannaichang@hotmail.com (N.Y.) \\ * Correspondence: huanglei_nudt@163.com
}

Received: 11 March 2019; Accepted: 15 April 2019; Published: 17 April 2019

\begin{abstract}
A novel method to design a wideband substrate integrated waveguide (SIW) bandpass filter (BPF) with compact size, wide stopband and high selectivity is presented. In this method some unique electromagnetic band-gap (EBG) cells are periodically etched on the top layer of SIW to realize a wide passband propagating below the equivalent waveguide cutoff frequency. By changing the configuration of EBG cells, undesired harmonics in upper stopband can be suppressed and a wideband BPF with wide stopband can be obtained. By symmetrically loading two complementary split ring resonators (CSRRs) on the tapered gradient lines of the input/output ports, a transmission zero near the passband can be introduced, and it makes the frequency selectivity of upper sideband improve significantly. As a verification, a wideband SIW BPF with a $3.02 \mathrm{GHz}$ absolute bandwidth (ABW) and a $64.7 \%$ fractional bandwidth (FBW) centered at $4.67 \mathrm{GHz}$ is designed, simulated, manufactured, and measured. The results of the experiment and simulation are in good agreement.
\end{abstract}

Keywords: wideband; bandpass filter; substrate integrated waveguide (SIW); compact microstrip resonant cell (CMRC); complementary split ring resonator (CSRR); transmission zero (TZ)

\section{Introduction}

Filters play a very important role in many microwave and radio frequency (RF) applications. Burgeoning applications such as wireless communication systems continue to challenge microwave/RF filters with even more stringent requirements [1]. At the same time, with the rapid development of the fifth-generation (5G) mobile communication technology, wideband wireless communication systems are also being widely developed and applied, because of their high transmission rate, low power consumption, high security and anti-interference ability. Therefore, wideband bandpass filters (BPFs) have received tremendous attention as a key and essential passive component in wideband wireless communication systems. Recently various methods and techniques for the design of wideband BPFs are constantly being presented. However, up until now the design of the wideband BPFs with compact size, high selectivity, low cost, wide stopband and high power capacity is still a challenging task. Traditional transmission line technologies, whether planar or non-planar, cannot fully meet all of these requirements. Fortunately, a structure called a wideband substrate integrated waveguide (SIW), which combines the characteristics of both planar and non-planar circuits, has emerged and developed extensively due to its potential applications to the design of excellent filters [2]. However, the typical bandwidths of these filters based on SIW technology are very narrow, generally less than $20 \%$ or even $15 \%$ [3]. In [4], a modified SIW zigzag filter topology is proposed to achieve the wideband feature. But the circuit size is large for engineering applications and its fractional bandwidth is still only $28 \%$. In order to improve the compactness of a wideband SIW filter, the ridge half-mode SIW (RHMSIW) structure is presented in [5], which provides the implementation to design wideband filter of quite a 
small size. Although the aforementioned improvement is substantial, the RHMSIW BPF has narrow stopband region and low selectivity. In [6], a SIW BPF with wide stopband is proposed by utilizing the defected ground structure (DGS). The wideband and high skirt selectivity features can be achieved. But the structure can hardly be used in high frequency systems due to its poor anti-interference ability and its bottom has to be kept away from other metals. In [7], the cross coupling technique is exploited, which can generate some transmission zeros (TZs) to improve stopband performance. Nevertheless, it is difficult to adjust the coupling coefficients between resonators and the stopband is also limited $2 f_{0}$. In particular, all of the filters mentioned above have a very high operating frequency, and few reports indicate that SIW technology can be applied to the low frequency domain.

This paper presents a novel method to design an excellent wideband BPF by combining SIW with some advanced technologies. Compared with other traditional structures, the proposed filter can work in a lower frequency with a more compact size. It can also generate multiple tunable TZs to obtain a good stopband performance. Moreover, the bandwidth and center frequency of the proposed filter can be controlled arbitrarily by adjusting different parameters. The most important point is that the designed wideband BPF has a perfect signal fidelity and a good time-domain behavior. As a verification, a wideband SIW BPF with wide stopband and high selectivity is designed, simulated, fabricated, and experimented. Detailed working mechanisms and design methods are explained as follows.

\section{Design Method and Analysis}

Figure 1a shows the three-dimensional (3D) geometry of the proposed wideband SIW BPF, while the physical parameters are depicted in Figure 1b. The filter is directly excited by two 50-ohm microstrip feed lines, which are connected to the SIW by two tapered gradient lines. The smooth transition can convert the quasi transverse electric and magnetic field (TEM) mode transmitted in microstriplin into a waveguide mode to ensure a field matching between microstrip and SIW. Three periodically arranged and two identical complementary split ring resonators (CSRRs) are etched on the surface of the SIW and tapered gradient lines, respectively. The ground is covered completely by a metal, which is not be shown in Figure 1. The dielectric substrate used in the design is a Rogers $4350 \mathrm{~B}$ which has a relative dielectric constant $\varepsilon_{\mathrm{r}}=3.66$, a loss tangent $\tan \sigma=0.0037$, and its thickness is set to $0.508 \mathrm{~mm}$.

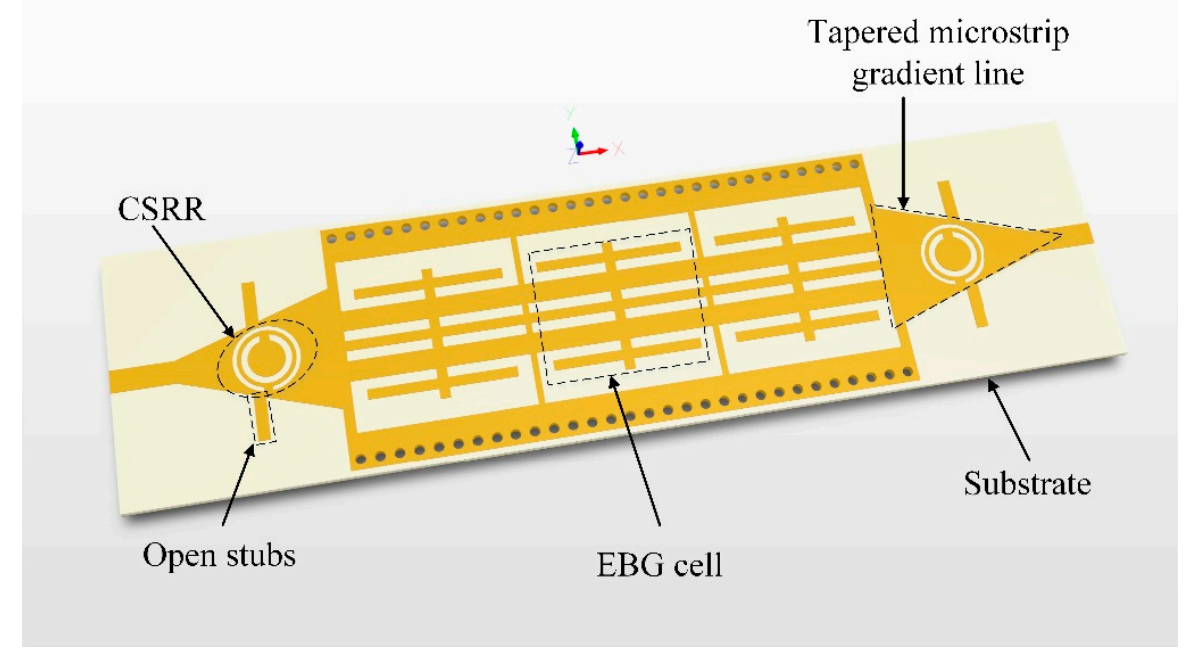

(a)

Figure 1. Cont. 


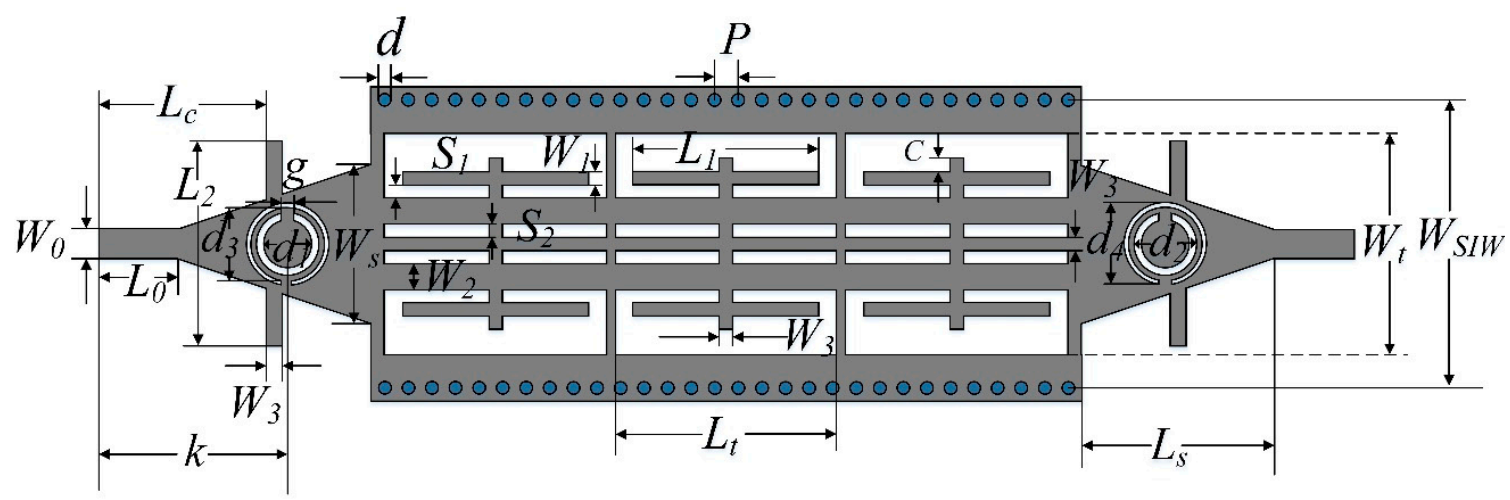

(b)

Figure 1. Structure of proposed bandpass filter (BPF). (a) Three-dimensional (3D) view. (b) Planar view.

The SIW, as a most basic element in the proposed BPF, is a kind of special waveguide loaded by a dielectric substrate. It has similar field distribution and transmission characteristics with traditional rectangular waveguide (RWG). If the electromagnetic waves are lower than the cutoff frequency, it will not be able to propagate in the SIW. So the SIW is used to provide a highpass characteristic in this design. Referring to the theory of RWG, the cutoff frequency of the SIW can be calculated by the following equation [8]:

$$
f_{c m 0}=\frac{c}{2 \sqrt{\varepsilon_{r} \mu_{r}}} \sqrt{\frac{m^{2}}{\left(W_{S I W}-\frac{d^{2}}{0.95 p}\right)^{2}}}
$$

where $c$ is the speed of light in vacuum, $\varepsilon_{r}$ is relative dielectric constant and $\mu_{r}$ is relative permeability of the used substrate, $m$ is the number of half waves propagating in the $x$-axis, $W_{S I W}$ represents the center distance between the two rows of metallized via holes, $d$ is the diameter of metallic vias and $p$ is the center spacing between adjacent via-holes.

For a lossless transmission line, the propagation constant is $\beta=\omega \sqrt{\mathrm{LC}}$. According to the transmission line theory, increasing distributed shunt capacitance $C$ and series inductance $L$ (i.e., high propagation constant) can make the transmission line behave with slow-wave characteristics. If the increase of $\mathrm{C}$ and $\mathrm{L}$ is discontinuous, the resonance between $\mathrm{L}$ and $\mathrm{C}$ will cause frequency-dependent reflection, forming bandstop characteristics. The adopted EBG cells consist of symmetrically arranged thin lines. Obviously, the horizontal thin lines increase the series inductance while the gap between the lines increase the shunt capacitance. When the EBG cells are connected periodically, it can be considered as a simple L-C parallel resonant circuit which can produce a stopband with a certain width. With the aid of full-wave simulator High Frequency Structure Simulator (HFSS) the frequency responses for the periodic EBG cells are shown in Figure 2. It can be clearly found that the periodic EBG cells exhibits a wide passband along with four transmission poles (TPs) at low frequencies. Therefore, a wide bandpass filter can be realized by combining the periodic EBG cells into the SIW. In order to independently control the lower and upper cutoff frequency, three EBG cells are periodically etched in the rectangular cutouts on the top metal surface of the SIW. The simulated frequency responses for the combination of SIW and EBG cells is presented in Figure 3, which is compared with the transmission coefficients of the initial SIW with the same dimensions. In fact, the proposed wideband BPF can essentially be considered as a cascade of a highpass filter and a bandstop filter. Lower and upper cutoff frequency are determined by SIW and EBG cells, respectively. The simulated results show that the lower cutoff frequency of the initial BPF is shifted from $9.36 \mathrm{GHz}$ to $3.51 \mathrm{GHz}$, which is equivalent to a $62 \%$ reduction in the size of the initial SIW. Strictly speaking, the slow-wave properties of periodic EBG cells lead to a substantial miniaturization on the size of the initial BPF. Moreover, the loaded EBG cells are also very useful to manipulate the magnitude and phase of the different harmonics to obtain the improved performance. In this design, the length of $L_{1}$ is tuned to make the higher harmonic poles disappear. A prototype of EBG-loaded SIW filter and its simulated results with different length $L_{1}$ are 
shown in Figure 4. Apparently it can be seen, when $L_{1}=4.5 \mathrm{~mm}$, that a transmission pole appears at 11.2 GHz, while a transmission zero (TZ) near the spurious band is located at $12.2 \mathrm{GHz}$. Changing the length of $L_{1}$ causes the TZ to move. In order to eliminate the undesired harmonic, the TZ has to be moved to the low frequency until it coincides with the transmission pole. As expected, when $L_{1}=5.7 \mathrm{~mm}$, the transmission pole disappears and the filter has a wider stopband of up to $13.36 \mathrm{GHz}$ with more than $20 \mathrm{~dB}$ suppression. Also it is noted that changing the length $L_{1}$ has no obvious effect on the lower cutoff frequency. So the proposed structure has a good independence in tuning the lower and upper cutoff frequency.

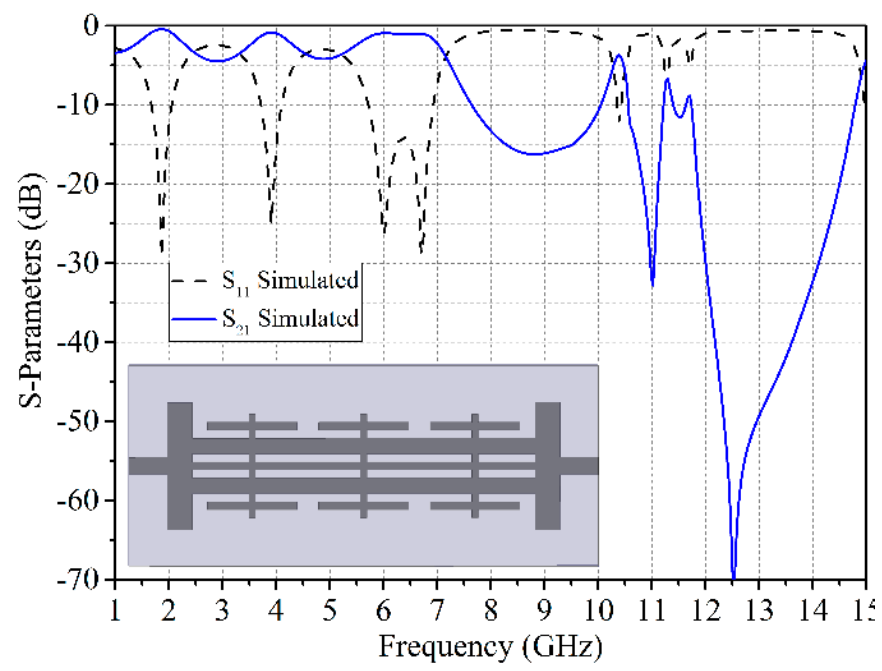

Figure 2. Simulated response of the periodic electromagnetic band-gap (EBG) cells.

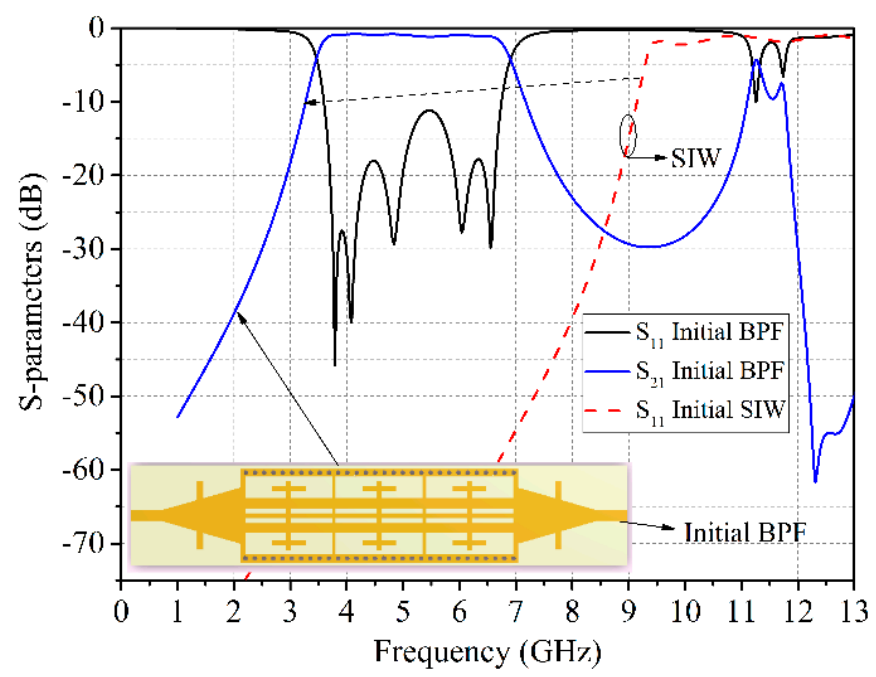

Figure 3. Wideband frequency responses of the initial BPF compared with the corresponding simulated substrate integrated waveguide (SIW) results. The initial physical dimensions are as follows (cells: $\mathrm{mm}): d=0.5, P=0.9, W_{0}=1.1, W_{1}=0.5, W_{2}=1, W_{3}=0.6, W_{S I W}=9, W_{s}=5.6, W_{t}=8, L_{0}=3$, $L_{3}=6.8, L_{c}=6.4, L_{s}=7.8, L_{t}=8.5, S_{1}=0.7, S_{2}=0.5, g=0.5, C=0.5$. 


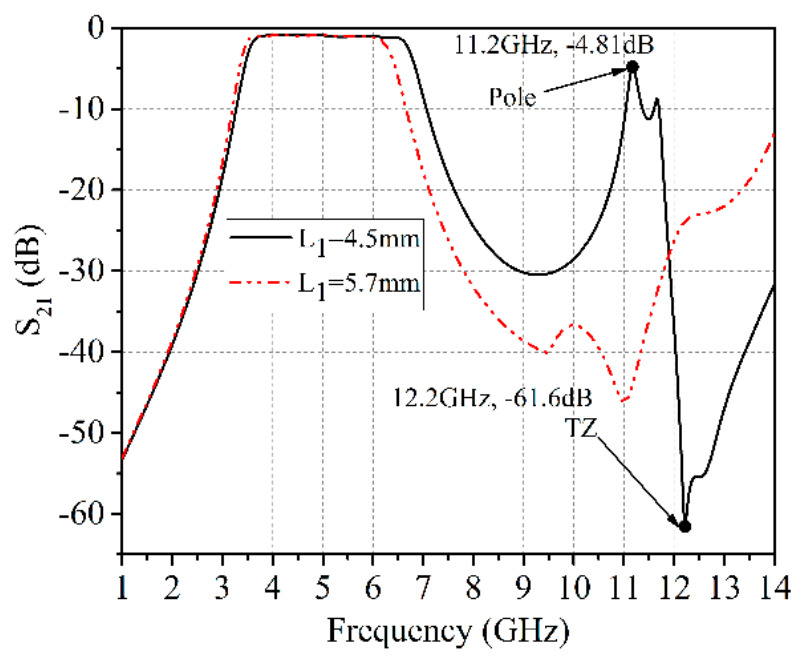

Figure 4. Variations of the filter responses versus $L_{1}$.

For this design, finding an effective way to improve the out-of-band selectivity of the proposed filter is critical. It is well known that introducing some new frequency transmission zeros (TZs) near the passband is a good choice to obtain a better selectivity compared with increasing the order of the filter which leads to a larger circuit size and a higher insertion loss (IL) [9]. As studied in [10] and [11], due to the effects of negative permittivity near the resonant frequency, a CSRR can be viewed as electric dipoles and can be excitied by an axial electric field. From previously reported literature [12-14], it is shown that, for the CSRR-loaded waveguide, the CSRRs can generate a stopband above the waveguide cutoff frequency. All the applications mentioned above indicate that CSRR can be used as a good candidate to improve the selectivity of the wideband BPF. With respect to the direction of wave propagation, a pair of identical circular CSRRs are etched on the linearly tapered microstrip gradient lines of the input and output ports in this design as in Figure 1, so that they can produce a transmission zero near the upper stopband. To take into account the passband and stopband in a balanced way, the finial geometrical parameters and the comparison of simulated frequency response results of the SIW BPF with and without the CSRRs are depicted in Figure 5. This shows that an additional transmission zero at $8.11 \mathrm{GHz}$ with $-65 \mathrm{~dB}$ attenuation level is created by CSRRs, which leads to a steep upper side transition and greatly improves the selectivity of the wideband SIW BPF. In addition, the introduced CSRRs can also add a new transmission pole at $5.44 \mathrm{GHz}$, which improves the transmission characteristics of high frequency in the passband of the BPF. 


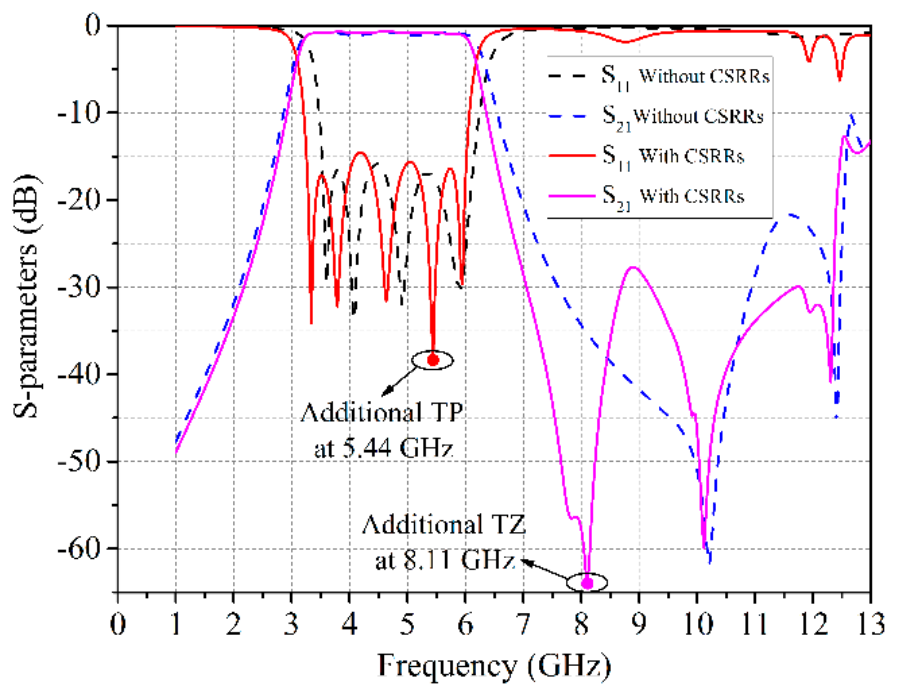

Figure 5. Simulated results of the BPF with and without complementary split ring resonators (CSRRs). The finial geometrical parameters are (cells: mm): $d=0.5, P=0.9, W_{0}=1.1, W_{1}=0.5, W_{2}=1$, $W_{3}=0.5, W_{S I W}=11, W_{s}=6, W_{t}=8.5, L_{0}=3, L_{1}=7.1, L_{2}=7.8, L_{c}=6.4, L_{s}=7.8, L_{t}=8.5$, $S_{1}=0.5, S_{2}=0.5, g=0.5, C=0.5, d_{1}=1.8, d_{2}=2.4, d_{3}=2.8, d_{4}=3.2, k=7.2$.

To better clarify the principle of CSRR generating TZ, the electric field magnitude distribution of the SIW BPF at $8.11 \mathrm{GHz}$ is depicted in Figure 6. As can be seen from the figure, the strong electric field distribution is mainly concentrated between the metal strip between the two slots and central conductive block of the CSRR at the input port. The electromagnetic wave with a frequency of $8.11 \mathrm{GHz}$ cannot propagate to the output port, resulting in a transmission zero.

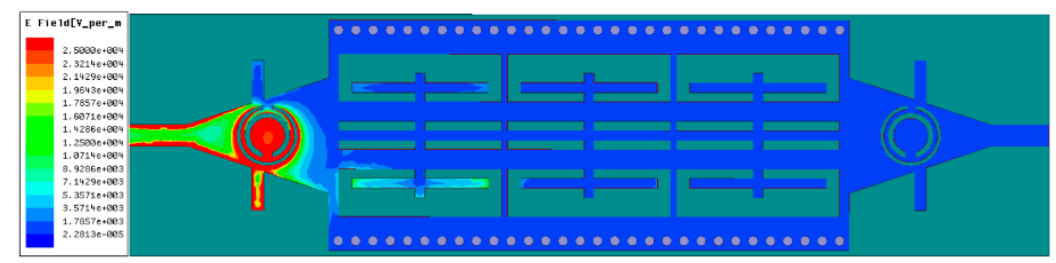

Figure 6. Electric field magnitude distribution in the BPF at $8.11 \mathrm{GHz}$.

Figure 7 presents an equivalent circuit for the CSRRs-loaded SIW. In this lumped-element circuit model, the losses caused by the dielectric substrate is ignored. The SIW can be regarded as a common two-transmission line loaded with two rows of short-circuited vias which can be modeled as the inductance $L_{d}$. The CSRR is modeled by means of the shunt-connected resonant tank formed by the inductance $L_{c}$ and the capacitance $C_{c}$. $L_{s}$ represents the inductive connection mainly through the split of the outer ring between the tapered microstrip gradient lines and the ring resonators [8]. In advance, the characteristics of the equivalent circuit for CSRR is analyzed using electronic design automation (EDA) software ADS (2016.01, Keysight Technologies, Santa Rosa, United States, 2015). Simulated results of the equivalent circuit are presented in Figure 8. The reflection coefficient $S_{11}$ and the transmission coefficient $S_{21}$ are represented by the blue dashed line and red solid line, respectively. It is clearly seen that the equivalent circuit can generate a TZ and a TP, which are consistent with the results of the BPF with CSRRs simulated by HFSS. According to knowledge of microwave circuits, the equivalent circuit for the CSRRs-loaded SIW gives a transmission zero frequency at

$$
f_{T Z}=\frac{1}{2 \pi} \sqrt{\frac{L_{c}+L_{s}}{L_{s} L_{c} C_{c}}}
$$


The transmission pole frequency can be obtained as follows:

$$
f_{T P}=\frac{1}{2 \pi} \sqrt{\frac{1}{L_{c} C_{c}}}
$$

In order to prove the validity of the frequency response, which is generated by the equivalent circuit model, an extensive comparison including both the result simulated by equivalent circuit model (marked as A), the transmission coefficient $S_{21}$ of the BPF without CSRRs (marked as B) and the sum of $A$ and $B$ is presented in Figure 9, which is compared with the simulated $S_{21}$ of the BPF with CSRRs. It is easy to find that the curve of $A+B$ can fit the simulated $S_{21}$ of the BPF with CSRRs, which verifies our proposed equivalent circuit model.

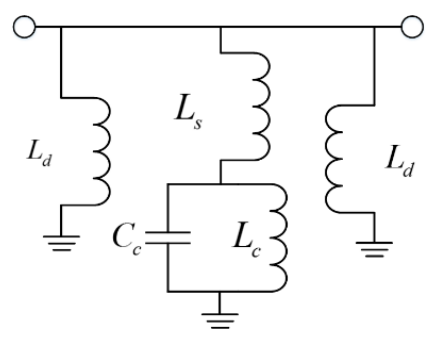

Figure 7. Equivalent circuit for the CSRR-loaded substrate integrated waveguide (SIW).

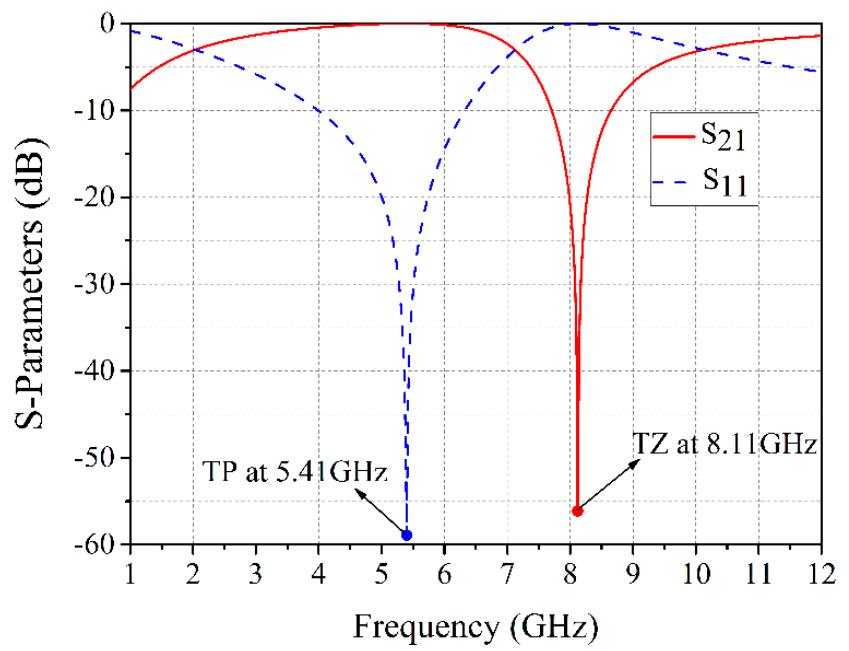

Figure 8. Frequency responses of equivalent circuit for CSRRs-loaded SIW. The electrical parameters shown in Figure 7 are $L_{s}=0.79 \mathrm{nH}, L_{c}=1 \mathrm{nH}, C_{c}=0.87 \mathrm{pF}$.

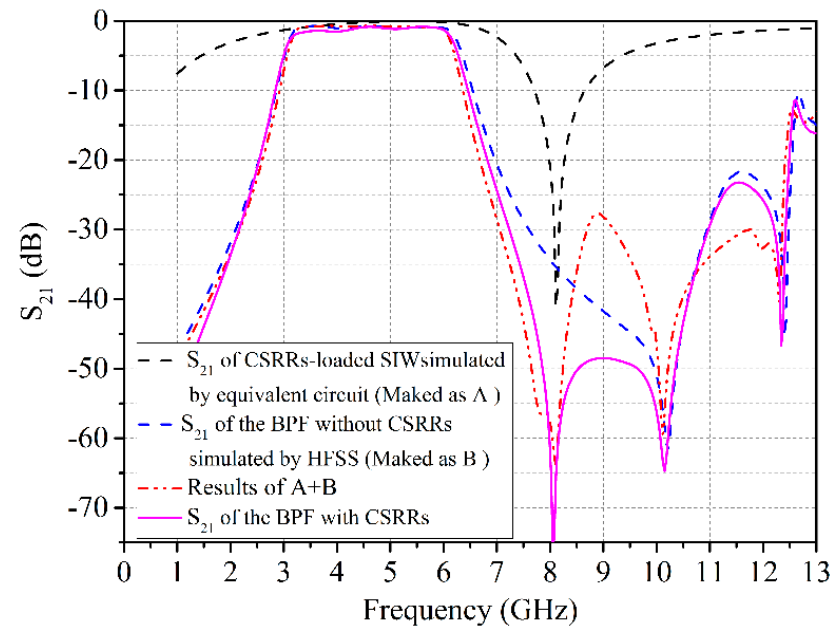

Figure 9. Simulated verification of equivalent circuit model. 
It should be noted that the additional TZ near the passband is mainly determined by the size of the CSRRs and it can be controlled independently. Changing the dimensions of the CSRRs and the different simulated frequency responses from full-wave simulator HFSS are shown in Figure 10 Obviously, it can be confirmed that the frequency of the additional $\mathrm{TZ}$ can be controlled individually by appropriately adjusting the size of the CSRRs, and the change in the dimensions of the CSRRs hardly affect the transmission characteristics at other frequencies of the proposed filter.

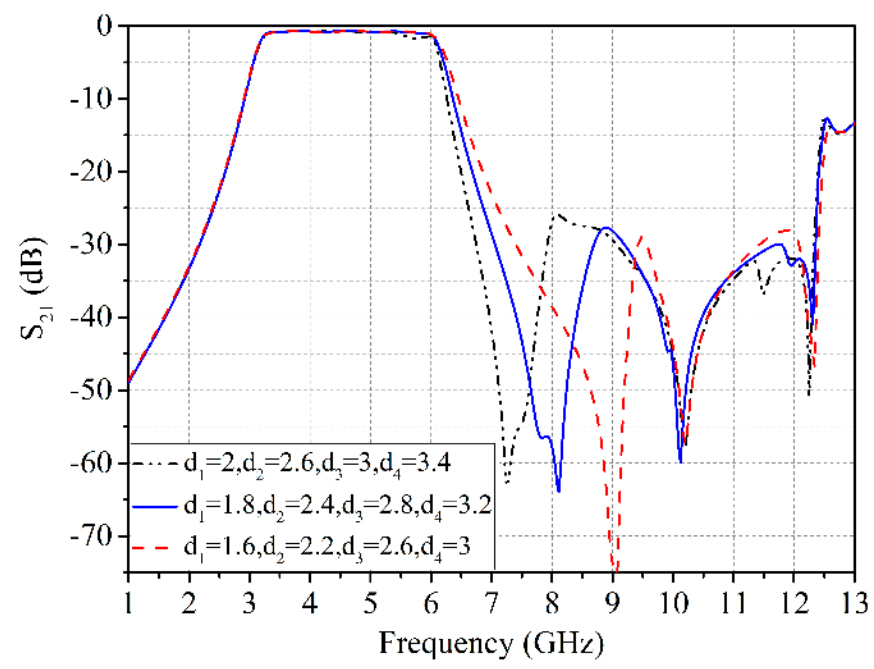

Figure 10. Simulated results for the proposed BPF with varied complementary split ring resonator (CSRR) sizes.

In wideband wireless communication applications, the filter is required to have a good signal fidelity capability, which cannot be obtained from its frequency-domain analysis. So an investigation of the filter time-domain behavior has to be performed. With the help of 3D electromagnetic simulator CST (2016.00, Computer Simulation Technology GmbH, Dumstadt, Germany, 2015), the filter is excited by a Gaussian pulse with a frequency of $3.16 \mathrm{GHz}$ to $6.18 \mathrm{GHz}$. The as-simulated filter impulse response is plotted in Figure 11. The Gaussian pulse is transmitted through the proposed BPF with only a delay in time, and there is hardly any change in its shape. As a verification, a sine step signal that varies from $3.16 \mathrm{GHz}$ to $6.18 \mathrm{GHz}$ is used to excite the filter, the response curve is shown Figure 12. The same conclusion can be obtained. Hence, the filter has a perfect signal fidelity and a good time-domain behavior.

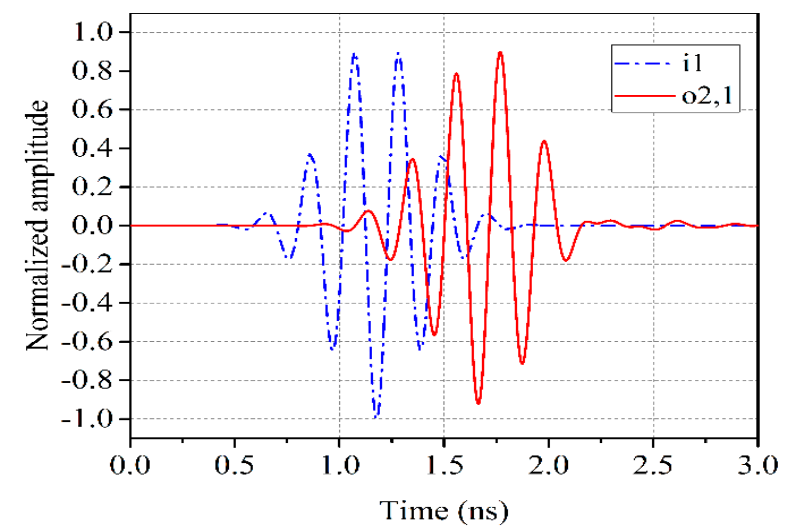

Figure 11. Normalized filter Gaussian impulse response. 


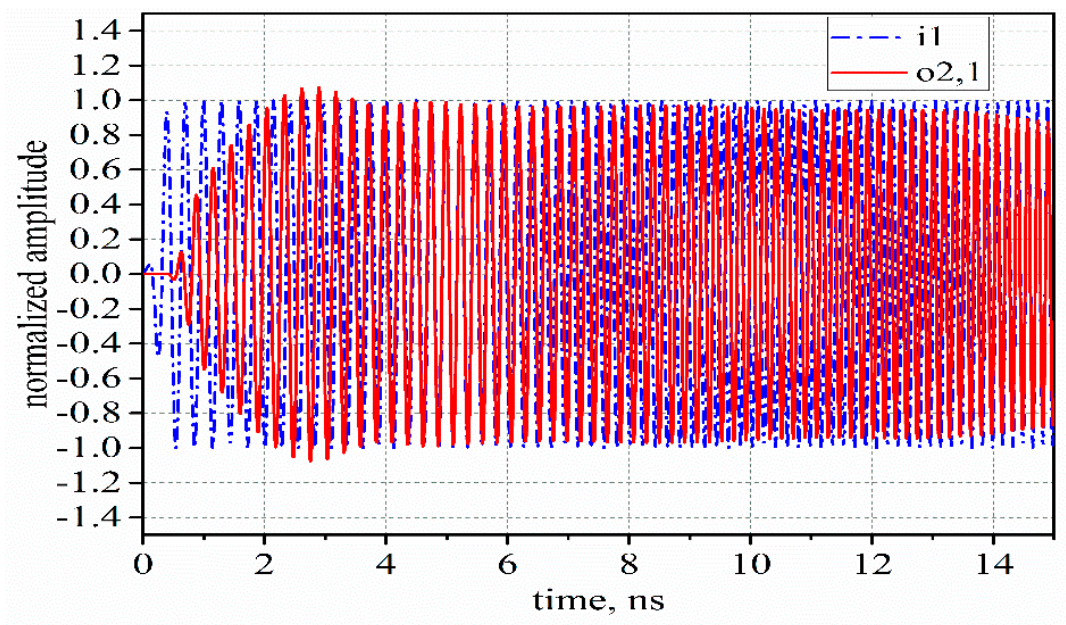

Figure 12. Normalized filter sine step impulse response.

\section{Measured Results and Discussion}

A compact wideband SIW BPF with wide stopband and high selectivity is designed and fabricated on a $0.508 \mathrm{~mm}$-thick Rogers $4350 \mathrm{~B}$ substrate $\left(\varepsilon_{\mathrm{r}}=3.66, \tan \delta=0.0037\right)$ using a standard single-side printed circuit board (PCB) process. Its photograph and the test fixture with flange joints are given in Figure 13. The total circuit dimension excluding two $3 \mathrm{~mm}$ feeding lines is $12.6 \mathrm{~mm} \times 47.9 \mathrm{~mm}$, corresponding to $0.31 \lambda_{g} \times 1.18 \lambda_{g}$, where $\lambda_{g}$ is the guided wavelength in the substrate at center frequency of $4.67 \mathrm{GHz}$. To validate the above design approach, the fabricated BPF is measured on a vector network analyzer Agilent N5230 (Agilent Technologies, California, U.S.) series. Figure 14 shows the simulated (dashed line) and measured (solid line) results. There are some slight differences between the measured and the simulated results due to machining errors and imperfect soldering processes. The measured $3 \mathrm{~dB}$ passband is from $3.16 \mathrm{GHz}$ to $6.18 \mathrm{GHz}$ with a fractional bandwidth of $64.7 \%$. The measured in-band return loss is larger than $12.1 \mathrm{~dB}$, while the minimum insertion loss is $1.0 \mathrm{~dB}$. The in-band fluctuation as observed in the inset of Figure 14 is less than $0.2 \mathrm{~dB}$. It is worth mentioning that the measured S-parameters include two vertical transitions and sub-miniature-A (SMA) connectors welded on two ends of the designed BPF for connecting input/output ports. Hence, the minimum insertion loss of the filter is definitely lower than $1 \mathrm{~dB}$. Four transition zeroes are located at $8.05 \mathrm{GHz}, 10.53 \mathrm{GHz}, 12.12 \mathrm{GHz}$ and $12.7 \mathrm{GHz}$, respectively, which improve the selectivity and also preserve a good upper stopband performance with attenuation level higher than $20 \mathrm{~dB}$ from $6.76 \mathrm{GHz}$ to $12.82 \mathrm{GHz}$ or $2.75 f_{0}$.

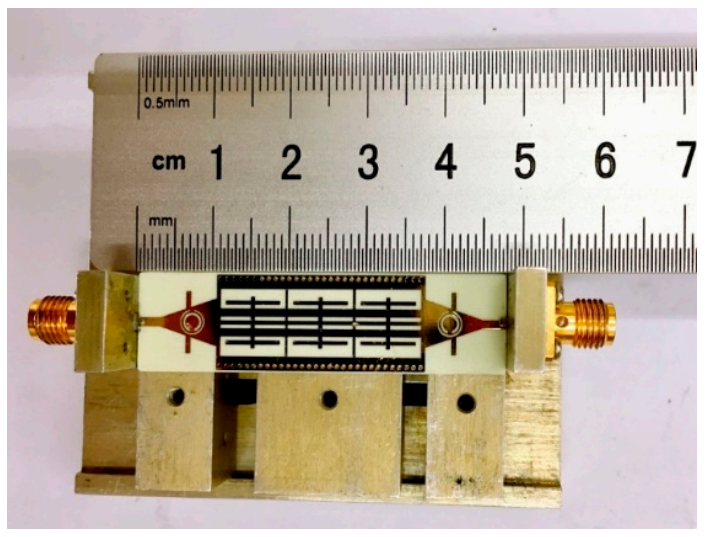

Figure 13. Photograph of the fabricated BPF and test fixture. 


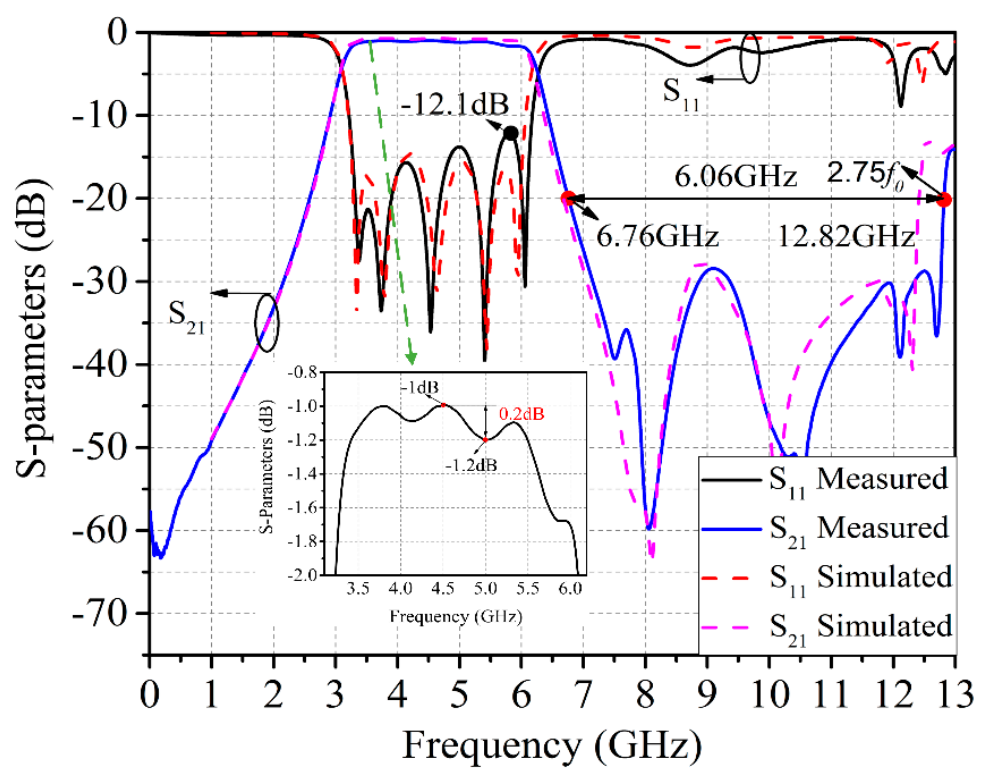

Figure 14. Measured and simulated results of the proposed wideband BPF.

Table 1 depicts a comparison among the proposed BPF and some published BPFs with SIW structure. It is important to note that the parameter $\mathrm{BW}_{20 \mathrm{~dB}} / \mathrm{BW}_{3 \mathrm{~dB}}$ represents the quality of selectivity. The smaller the value of $\mathrm{BW}_{20 \mathrm{~dB}} / \mathrm{BW}_{3 \mathrm{~dB}}$, the better the selectivity. As shown in Table 1 , the proposed SIW BPF exhibits better performance, including greater fractional bandwidth, better frequency selectivity, wider stopband, lower insertion loss, and smaller size. All in all, the filter proposed in this paper outperforms the quoted filters.

Table 1. Comparison with other published substrate integrated waveguide (SIW) bandpass filters (BPFs).

\begin{tabular}{ccccccc}
\hline Ref. & $\mathbf{C F}(\mathbf{G H z})$ & FBW $_{\mathbf{3 d B}} \mathbf{( \% )}$ & $\mathbf{I L} \mathbf{( d B )}$ & $\mathbf{B W}_{\mathbf{2 0 d B}} / \mathbf{B W}_{\mathbf{3 d B}}$ & ${ }^{*}$ Rej. $(\mathbf{d B}) / \mathbf{S . B}$. & Size $\left(\lambda_{g}^{2}\right)$ \\
\hline$[3]$ & 8.5 & 42 & 1.1 & 1.22 & $20 / 1.8 f_{0}$ & 0.79 \\
\hline$[4]$ & 7.26 & 27.8 & 1.5 & - & $25 / 1.4 f_{0}$ & 4.7 \\
\hline$[15]$ & 7.51 & 59 & 1.5 & 1.37 & $20 / 1.8 f_{0}$ & 2.71 \\
\hline$[16]$ & 5.75 & 1.8 & 3.6 & 1.95 & $20 / 2 f_{0}$ & 0.74 \\
\hline$[17]$ & 12.25 & 61.5 & 1.55 & - & - & 1.82 \\
\hline This work & 4.67 & 64.7 & 1.0 & 1.38 & $20 / 2.7 f_{0}$ & 0.36 \\
\hline \multicolumn{7}{c}{ Rej. (dB)/S.B.: Rejection (dB)/Stopband. }
\end{tabular}

\section{Conclusions}

In this paper, a compact wideband SIW BPF with wide stopband and high selectivity has been investigated and designed. Detailed design methods and steps have also been presented. This BPF allows the effective combination of the SIW with EBG cells and CSRRs in a compact form. All parts of the proposed structure are independent, and the resonant frequency of every part mainly depends on their own size, thus providing us with a good freedom to control the operating center frequency and bandwidth arbitrarily. As a verification, a prototype operated at $3.16 \mathrm{GHz}$ to $6.18 \mathrm{GHz}$ has been designed, simulated, manufactured, and experimented with. Good agreement can be observed between simulated and measured results. In particular, the proposed filter has a perfect signal fidelity and a good time-domain behavior. It can be widely applied in various broadband wireless communication systems. 
Author Contributions: Conceptualization, L.H.; Data curation, L.H.; Formal analysis, L.H.; Funding acquisition, N.Y.; Investigation, L.H.; Methodology, L.H.; Project administration, N.Y.; Resources, N.Y.; Supervision, N.Y.; Visualization, L.H.; Writing - original draft, L.H.; Writing - review and editing, L.H.

Funding: This research received no external funding.

Acknowledgments: I am very grateful to Naichang Yuan's guidance and help in my research.

Conflicts of Interest: All authors declare no conflict of interest.

\section{References}

1. Grine, F.; Djerafi, T.; Benhabiles, M.T.; Wu, K.; Riabi, M.L. High-Q Substrate Integrated Waveguide Resonator Filter With Dielectric Loading. IEEE Access 2017, 5, 12526-12532. [CrossRef]

2. Wu, Y.-L.; Chen, Y.-Q.; Jiao, L.-X.; Liu, Y.-X.; Ghassemlooy, Z. Dual-band dual-mode substrate intergrated waveguide filters with in dependently reconfigurable TE101 resonant mode. Sci. Rep. 2016, 6, 31922. [CrossRef] [PubMed]

3. Chen, R.S.; Wong, S.-W.; Zhu, L.; Chu, Q.-X. Wideband Bandpass Filter Using U-Slotted Substrate Integrated Waveguide (SIW) Cavities. IEEE Microwave Wireless Compon. Lett. 2015, 25, 1-3. [CrossRef]

4. Mira, F.; Mateu, J.; Cogollos, S.; Boria, V.E. Design of ultra-wideband substrate integrated waveguide (SIW) filters in ZigZag toplogy. IEEE Microwave Wireless Compon. Lett. 2009, 19, 281-283. [CrossRef]

5. Huang, L.; Cha, H.; Li, Y. Compact Wideband Ridge Half-Mode Substrate Integrated Waveguide Filters. IEEE Trans. Microwave Theory Tech. 2016, 64, 3568-3579. [CrossRef]

6. Kurudere, S.; Erturk, V.B. Novel SIW based interdigital bandpass filter with harmonic suppression. In Proceedings of the 44th European Microwave Conference, Rome, Italy, 6-9 October 2014.

7. Zhu, F.; Chen, J.; Hong, W.; Wu, K. Wide stopband substrate integrated waveguide filter using corner cavities. Electron. Lett. 2013, 49, 50-52. [CrossRef]

8. Moitra, S.; Bhowmik, P.S. Modelling and analysis of Substrate Integrated Waveguide (SIW) and half-mode SIW (HMSIW) band-pass filter using reactive longitudinal periodic structures. AEU Int. J. Electron. C 2016, 70, 1593-1600. [CrossRef]

9. Li, M.; Chen, C.; Chen, W. Miniaturized Dual-Band Filter Using Dual-Capacitively Loaded SIW Cavities. IEEE Microwave Wireless Compon. Lett. 2017, 27, 1-3. [CrossRef]

10. Azad, A.R.; Mohan, A. Substrate Integrated Waveguide Dual-Band and Wide-Stopband Bandpass Filters. IEEE Microwave Wireless Compon. Lett. 2018, 28, 660-662. [CrossRef]

11. Wu, Y.; Zhuang, Z.; Liu, Y.; Deng, L.; Ghassemlooy, Z. Wideband Filtering Power Divider with Ultra-Wideband Harmonic Suppression and Isolation. IEEE Access 2016, 4, 6876-6882. [CrossRef]

12. Dong, Y.D.; Yang, T.; Itoh, T. Substrate Integrated Waveguide Loaded by Complementary Split-Ring Resonators and Its Applications to Miniaturized Waveguide Filters. IEEE Trans. Microwave Theory Tech. 2009, 57, 2211-2223. [CrossRef]

13. Liu, Z.; Xiao, G.B.; Zhu, L. Triple-mode bandpass filters on CSRR-loaded substrate integrated waveguide caviyies. IEEE Trans. Microwave Theory Tech. 2016, 6, 1099-1105.

14. Zhang, Q.-L.; Yin, W.-Y.; He, S.; Wu, L.-S. Compact Substrate Integrated Waveguide (SIW) Bandpass Filter With Complementary Split-Ring Resonators (CSRRs). IEEE Microwave Wireless Compon. Lett. 2010, 20, 426-428. [CrossRef]

15. Zheng, C.; Xu, F. Compact bandpass filter based on one-third equilateral triangular resonator of substrate integrated waveguide. Electron. Lett. 2015, 51, 1505-1507. [CrossRef]

16. Sirci, S.; Soriano, M.A.S.; Martinez, J.D.; Boria, V.E.; Gentili, F.; Bosch, W.; sorrentino, R. Design and multiphysiscs analysis of direct and cross-coupled SIW combline filters using electric and magnetic couplings. IEEE Trans. Microwave Theory Tech. 2015, 63, 4341-4354. [CrossRef]

17. Hao, Z.-C.; Hong, W.; Chen, J.-X.; Chen, X.-P.; Wu, K. Compact super-wide bandpass substrate integrated waveguide (SIW) filters. IEEE Trans. Microwave Theory Tech. 2005, 53, 2968-2977. 